\title{
Medical Effects of Ionizing Radiation
}

\author{
F.A. Mettler, Jr. and A.C. Upton \\ Philadelphia, PA: Saunders, 2008, 564 pages, \$207
}

This third edition of a landmark textbook follows the first edition by $22 \mathrm{y}$ and the second edition by $13 \mathrm{y}$. Consisting of 476 pages of text and 50 pages of useful appendices, this edition is intended to be a resource for nuclear physicians and physicians of other related specialties, as well as for nurses and physicists working in related fields of nuclear imaging and therapy. As stated in the preface, the primary changes in the intervals between editions have included the increased magnitude of medical radiation exposure to the general population, molecular and cellular insights on potential mechanisms of low-dose effects, and information from epidemiologic studies on site-specific cancer risks. Information on radium and on psychologic aspects has been added, and the broader perspective on radiation effects has been significantly rewritten. Normal-tissue toxicity grading has also been added to the appendix.

This book is organized into 12 chapters. After the first 2 chapters on the basics of nuclear science, the authors describe radiation effects on genes, tissues, and organs, as well as carcinogenesis, in the next 4 chapters. The following 4 chapters concentrate on combination effects with chemotherapy, specific effects on the fetus, specific radionuclides, and the application of probabilities to understand potential risk to specific individuals. The book concludes with a chapter on the perception of radiation by the public and a chapter briefly discussing hormesis.

The book is well organized and well written, with a logical progression from section to section and from chapter to chapter. This book discusses the effects of high- and lowlevel radiation exposures, and the direct, carcinogenic, and genetic effects of ionizing radiation are discussed in detail. Animal data are not generally included since they are rarely applicable to humans without major assumptions. The information provided is succinct and up to date. The first 2 chapters, although succinct, are still able to convey adequate scientific understanding of atomic and molecular events that can also be understood in quantitative terms of dosimetry. Although the discussion of effects on organs may appear lengthy, that historic and current information is essential to understanding radiation effects on humans. On the other hand, many diagrams and figures are of low resolution and may be improved in the next edition. An extensive glossary and a collection of useful tables are appended.

Reading through this book, one will be reminded of many forgotten concepts and facets. In addition to the tables and figures, the appendices are useful for looking up dosimetry conversions and for comparing the effects of different radionuclides. In summary, this book is a handy resource that also presents radiation effects in an organized and logical manner. Practitioners of nuclear medicine and related disciplines should keep this book readily accessible, if not on their desk.

Franklin C. Wong

E. Edmund Kim*

${ }^{*}$ M.D. Anderson Cancer Center

1515 Holcombe Blvd., Unit 1264

Houston, TX 77030

E-mail:ekim@di.mdacc.tmc.edu 